\title{
On the surjectivity of Galois representations attached to elliptic curves over number fields
}

\author{
by \\ Álvaro Lozano-Robledo (Waterville, ME)
}

1. Surjectivity of a Galois representation. Let $K$ be a number field, fix $\bar{K}$, an algebraic closure of $K$, and let $j$ be transcendental over $K$. Let $E$ be an elliptic curve defined over the field $K(j)$ such that $j(E)=j$. Given a prime number $p \geq 7$, the natural action of $\operatorname{Gal}(\overline{K(j)} / \bar{K}(j))$ on the group of $p$-torsion points of $E$ induces a representation $\widetilde{\pi}_{E}: \operatorname{Gal}(\overline{K(j)} / \bar{K}(j)) \rightarrow$ $\operatorname{SL}\left(2, \mathbb{F}_{p}\right)$. The universal deformation of $\widetilde{\pi}_{E}$, with respect to certain ramification conditions (see [Roh], [Roh04]), is an epimorphism

$$
\pi_{E}: \operatorname{Gal}(\overline{K(j)} / \bar{K}(j)) \rightarrow \mathrm{SL}\left(2, \mathbb{Z}_{p}[[X]]\right) .
$$

Let $\widetilde{K}$ be the extension of $K$ generated by all roots of unity of $p$-power order. In [Roh00a], [Roh00b], D. E. Rohrlich showed that $\pi_{E}$ descends to an epimorphism

$$
\varrho_{E}: \operatorname{Gal}(\overline{K(j)} / \widetilde{K}(j)) \rightarrow \mathrm{SL}\left(2, \mathbb{Z}_{p}[[X]]\right) .
$$

Notice that $\varrho_{E}$ encapsulates arithmetic information which was not present in $\pi_{E}$.

Let $A$ be an elliptic curve defined over $K$ with $j$-invariant $j(A) \notin\{0,1728\}$ and suppose that $A$ coincides with the fiber of $E$ at $j=j(A)$. Choose a place $\sigma$ of $\overline{K(j)}$ extending the place $j=j(A)$ of $\widetilde{K}(j)$, and write $D$ and $I$ for the corresponding decomposition and inertia subgroups of $\operatorname{Gal}(\overline{K(j)} / \widetilde{K}(j))$. We "specialize" the representation $\varrho_{E}$ to $j=j(A)$ by restricting the map to the decomposition group $D$. By the ramification constraints of the universal deformation (see $\left[\right.$ Roh00b]), the map $\varrho_{E}$ is unramified outside $\{0,1728, \infty\}$, thus $\left.\varrho_{E}\right|_{D}$ factors through $D / I \cong \operatorname{Gal}(\bar{K} / \widetilde{K})$. We obtain a representation

$$
\varrho_{A}: \operatorname{Gal}(\bar{K} / \widetilde{K}) \rightarrow \operatorname{SL}\left(2, \mathbb{Z}_{p}[[X]]\right) .
$$

2000 Mathematics Subject Classification: Primary 11F80; Secondary 11G05.

Key words and phrases: p-adic Galois representations, deformations, surjectivity, elliptic curves. 
If we write $\bar{\varrho}_{A}: \operatorname{Gal}(\bar{K} / \widetilde{K}) \rightarrow \operatorname{SL}\left(2, \mathbb{Z}_{p}\right)$ for the representation determined up to equivalence by the natural action of $\operatorname{Gal}(\bar{K} / \widetilde{K})$ on the Tate module of $A$, then, by construction, $\varrho_{A}$ is a deformation of $\bar{\varrho}_{A}$, and in particular $\left.\varrho_{A}\right|_{X=0}=\bar{\varrho}_{A}$. The image of $\bar{\varrho}_{A}$, which has been characterized by M. Deuring [Deu53], [Deu58], J.-P. Serre [Ser72], J. Tate [ST68] and others, depends drastically on whether the elliptic curve $A$ has complex multiplication or not.

In light of the results of Deuring, Serre and Tate, one would naturally want to know how large is the image of the representation $\varrho_{A}$. Let

$$
\widetilde{\varrho}_{A}: \operatorname{Gal}(\bar{K} / \widetilde{K}) \rightarrow \mathrm{SL}\left(2, \mathbb{F}_{p}\right)
$$

be the representation induced by the Galois action on the points of order $p$ on $A$. In [Roh04], Rohrlich proved in the case $K=\mathbb{Q}$ that if $\widetilde{\varrho}_{A}$ is surjective and $\nu_{p}(j(A))=-1$ then $\varrho_{A}$ is surjective, where $\nu_{p}$ is the usual $p$-adic valuation on $\mathbb{Q}$. In this note we generalize Rohrlich's results to more general number fields.

Fix $\wp$, a prime of $K$ lying above a prime $p \geq 7$. We write $\nu_{\wp}$ for the standard $\wp$-adic valuation on $K$, so that, for a uniformizer $\pi$ of $\wp, \nu_{\wp}(\pi)=1$ and $\nu_{\wp}(p)=e$, where $e=e(\wp \mid p)$ is the ramification index.

THEOREM 1.1. If $\widetilde{\varrho}_{A}$ is surjective, $e$ is not divisible by $p-1, \nu_{\wp}(j(A))$ $=-t$ with $t \in \mathbb{N}, \operatorname{gcd}(p, t)=1$, and

$$
t<\frac{e p}{p-1}=e+\frac{e}{p-1}
$$

then $\varrho_{A}$ is surjective.

Proof. The strategy of the proof is the same as in [Roh04, proof of Theorem 1] (which shows the case $K=\mathbb{Q}$ ). We summarize it here and point out where the proof diverges for a number field $K$ as in the statement of Theorem 1.1.

It suffices to verify the surjectivity of the projective representation

$$
P \varrho_{A}: \operatorname{Gal}(\bar{K} / \widetilde{K}) \rightarrow \operatorname{PSL}(2, \Lambda)
$$

because the only subgroup of $\operatorname{SL}(2, \Lambda)$ with projective image $\operatorname{PSL}(2, \Lambda)$ is the full group $\mathrm{SL}(2, \Lambda)$. We similarly define projective maps $P \varrho_{E}$ and $P \bar{\varrho}_{A}$. By the definition of $\varrho_{A}$, in order to verify the surjectivity of $P \varrho_{A}$ it suffices to show that the image via $P \varrho_{E}$ of the decomposition group $D$ is the full group $\operatorname{PSL}(2, \Lambda)$.

The kernel of $\varrho_{E}$ determines a fixed field $\mathbf{L}$, in particular $\operatorname{Gal}(\mathbf{L} / \widetilde{K}(j)) \cong$ $\operatorname{PSL}\left(2, \mathbb{Z}_{p}[[X]]\right)$. For $i \geq 1$, let $\mathbf{L}_{i} \subseteq \mathbf{L}$ be the fixed field determined by the kernel of the reduction map

$$
\operatorname{Gal}(\mathbf{L} / \widetilde{K}(j)) \cong \operatorname{PSL}\left(2, \mathbb{Z}_{p}[[X]]\right) \rightarrow \operatorname{PSL}\left(2, \mathbb{Z}_{p}[[X]] /(p, X)^{i}\right) .
$$


Recall that we have chosen a place $\sigma$ of $\overline{K(j)}$ extending $j=j(A)$. Let $\ell_{\nu}$ be the residue class field of $\sigma \mid \mathbf{L}_{\nu}$, i.e. $\ell_{\nu}=\sigma\left(\mathbf{L}_{\nu}\right) \backslash\{\infty\}$.

A criterion of Boston ([Bos86, Prop. 2, p. 262]) reduces the problem to proving that the image of $D$ in $\operatorname{Gal}\left(\mathbf{L}_{2} / \widetilde{K}(j)\right)$ maps to all of $\operatorname{PSL}\left(2, \Lambda /(p, X)^{2}\right)$. Equivalently, one needs to show that $\left[\mathbf{L}_{2}: \widetilde{K}(j)\right]=\left[\ell_{2}: \widetilde{K}\right]$. Notice that the assumption on the surjectivity of $\widetilde{\varrho}_{A}$ implies that $\bar{\varrho}_{A}$ is surjective (see, for example, [Ser68, IV-23, Lemma 3]), and so is $P \bar{\varrho}_{A}$, the projectivization of $\bar{\varrho}_{A}$. It follows that $\left[\mathbf{L}_{1}: \widetilde{K}(j)\right]=\left[\ell_{1}: \widetilde{K}\right]$, therefore it suffices to prove that

$$
\left[\mathbf{L}_{2}: \mathbf{L}_{1}\right]=\left[\ell_{2}: \ell_{1}\right]
$$

1.1. Siegel functions. We follow the definitions established in [Roh04].

Definition 1.2. Let $p \geq 7$ be a prime and define $R=\mathbb{F}_{p}^{2} \backslash\{(0,0)\}$.

(1) $M$ is the set of all functions $m: R \rightarrow \mathbb{Z}$ with $m(r)=m(-r) . M$ is clearly a $\mathbb{Z}$-module.

(2) We write $N$ for the $\mathbb{Z}$-submodule of $M$ consisting of all those $m \in M$ that reduce modulo $p$ to a function defined by a homogeneous polynomial of degree two over $\mathbb{F}_{p}$.

Let $r \in R$ and let $s=\left(s_{1}, s_{2}\right) \in \mathbb{Z}^{2}$ be any lift of $r$, i.e. $s=\left(s_{1}, s_{2}\right) \equiv$ $r \bmod p$, and put $a=a_{s}=p^{-1}\left(s_{1}, s_{2}\right)$. Then the symbol $f_{r}$ represents any Siegel function $g_{a}^{12}$ (see [KL81, p. 29]). If $s \in \mathbb{Z}^{2}$ is replaced by another lift of $r$ then $f_{r}$ is multiplied by a $p$ th root of unity ([KL81, Remark on p. 30]), so the symbol $f_{r}(\tau)$ is only well defined up to $p$ th roots of unity. For $m \in M$ we also define the symbolic $m$ th power:

$$
f^{m}=\prod_{r \in R} f_{r}^{m(r)} .
$$

The key ingredient in the proof of Theorem 1.1 is given by the following result of Rohrlich ([Roh04, Theorem 2]).

TheORem 1.3. The extension $\mathbf{L}_{2} / \mathbf{L}_{1}$ is generated by pth roots of Siegel units. More precisely, $\mathbf{L}_{2}=\mathbf{L}_{1}\left(\left\{\left(f^{m}\right)^{1 / p}: m \in N\right\}\right)$.

Using the previous theorem, Rohrlich reduces the proof of (1) to the following local statement (see [Roh04, pp. 19, 20]; the argument is valid in our case, by simply replacing $\mathbb{Q}$ by $K)$. Since $\nu_{\wp}(j(A))=-t<0$ there is a unique Tate curve $B$ over $K_{\wp}$ with $j(B)=j(A)$. Suppose there is an $m \in N$ such that $\sigma\left(f^{m}\right)^{1 / p} \notin K_{\wp}\left(B\left[p^{\nu}\right]\right)$ for all sufficiently large $\nu \in \mathbb{N}$. Then equality (1) follows.

Let $\mathcal{O}_{\wp}$ be the ring on integers in $K_{\wp}$ and let $q$ be the unique element of $\pi \mathcal{O}_{\wp}$ such that $j(q)=j(B)$, where $\pi$, as before, is a uniformizer of $\wp$. Proposition 8 of [Roh04] can be generalized to: 
Proposition 1.4. There exists $m \in N$ such that:

$$
\sigma\left(f^{m}\right)=q^{\mu}(1-u q)\left(1-v q^{2}\right)=q^{\mu}(1+w q)
$$

with $\mu \in \mathbb{Z}, u, w \in \mathcal{O}_{\wp}^{\times}$, and $v \in \mathcal{O}_{\wp}$. In particular, $\sigma\left(f^{m}\right) \in K_{\wp}$.

The proof found in [Roh04] is valid without change. Let $f=f^{m}$ with $m$ as in the previous proposition. Hence, in order to finish the proof of Theorem 1.1, we need to show:

Proposition 1.5. Suppose that $v_{\wp}(j(A))=-t$ with $t \in \mathbb{N}$, $e$ is not divisible by $p-1, \operatorname{gcd}(p, t)=1$, and

$$
t<\frac{e p}{p-1}=e+\frac{e}{p-1} .
$$

Then $\sigma(f)^{1 / p} \notin K_{\wp}\left(B\left[p^{\nu}\right]\right)$ for all sufficiently large $\nu \in \mathbb{N}$.

Proof. It suffices to show that $\sigma(f)^{1 / p}$ has degree $p$ over $K_{\wp}\left(B\left[p^{\nu}\right]\right)$ for all sufficiently large $\nu$. Note that $K_{\wp}\left(B\left[p^{\nu}\right]\right)=K_{\wp}\left(\zeta, q^{1 / p^{\nu}}\right)$ where $\zeta$ is a primitive $p^{\nu}$ th root of unity (see [Lan87, Chapter 15, Theorem 3]).

Since $v_{\wp}(j(A))=-t$, we have $v_{\wp}(q)=t$ (and by assumption $\operatorname{gcd}(p, t)$ $=1)$. It follows that $\operatorname{gcd}\left(v_{\wp}(q), p^{\nu}\right)=1$ and the order of $q$ in $K_{\wp}^{\times} / K_{\wp}^{\times p^{\nu}}$ is $p^{\nu}$.

Recall that by Proposition 1.4 we can write $\sigma(f)$ as $q^{\mu}(1-u q)\left(1-v q^{2}\right)=$ $q^{\mu}(1+w q)$ with $\mu \in \mathbb{Z}, u, w \in \mathcal{O}_{\wp}^{\times}$, and $v \in \mathcal{O}_{\wp}$. We claim that $\alpha:=q^{-\mu} \sigma(f)$ has degree $p^{\nu}$ in $K_{\wp}^{\times} / K_{\wp}^{\times p^{\nu}}$. For suppose the contrary, i.e. $\alpha^{p^{\nu-1}}=\beta^{p^{\nu}}$ for some $\beta \in K_{\wp}$. Then $\beta^{p}=\xi \alpha$ with $\xi$ a $p^{\nu-1}$ th root of unity and $\xi=\beta^{p} \alpha^{-1}$ $\in K_{\wp}$. Since $K_{\wp}$ cannot contain nontrivial $p$ th roots of unity (or $p-1$ would divide $e$ ), it follows that $\xi=1$.

Hence $\alpha=\beta^{p}$. Let $\beta=1+b \pi$ for some $b \in \mathcal{O}_{\wp}, \pi$ a uniformizer for $\wp$. By the binomial theorem,

$$
(1+b \pi)^{p}=\sum_{h=0}^{p}\left(\begin{array}{l}
p \\
h
\end{array}\right) b^{h} \pi^{h},
$$

so the terms in $\beta^{p}-1$ have $\wp$-adic valuations in the list

$$
p\left(\nu_{\wp}(b)+1\right), \quad i\left(\nu_{\wp}(b)+1\right)+e \quad \text { with } 1 \leq i \leq p-1
$$

and the minimum nonzero valuation is either $p\left(\nu_{\wp}(b)+1\right)$ or $\nu_{\wp}(b)+1+e$ (and both cannot be equal, since that implies that $p-1$ divides $e$ ). This value must equal $t$ since we are assuming $\alpha=1+w q=\beta^{p}$, but $t$ is not divisible by $p$ by hypothesis, so the minimum valuation must be $t=\nu_{\wp}(b)+1+e$.

First suppose $t<e+1$. This implies that $\nu_{\wp}(b)<0$, which is contradictory since $b \in \mathcal{O}_{\wp}$. Otherwise $e+1 \leq t<e p /(p-1)$ and the fact that $\nu_{\wp}(b)+1+e<p\left(\nu_{\wp}(b)+1\right)$ implies that

$$
p>\frac{\nu_{\wp}(b)+1+e}{\nu_{\wp}(b)+1} .
$$


Substituting $\nu_{\wp}(b)=t-e-1$ we obtain $p>t /(t-e)$ and hence $t>$ $e p /(p-1)$ (since $t>e$ ), which contradicts our assumption on $t$. Therefore, we conclude that $\alpha$ is not a $p$ th power.

REMARK 1.6. Using the $\wp$-adic logarithm and exponential maps one can prove that if $\nu_{\wp}(\gamma)>e+e /(p-1)$ then $(1+\gamma)^{1 / p} \in K_{\wp}$. So the bound on $t$ in the theorem is best possible, at least for this method of proof.

Thus we have proved that the order of $\alpha$ in $K_{\wp}^{\times} / K_{\wp}^{\times p^{\nu}}$ is exactly $p^{\nu}$. Therefore, the subgroup of $K_{\wp}^{\times} / K_{\wp}^{\times p^{\nu}}$ generated by the cosets of $q$ and $\sigma(f)$ has order $p^{2 \nu}$.

Lemma 1.7. Let $L$ be a field with $\operatorname{char}(L)=0$, and let $\zeta$ be a primitive $p^{\nu}$ th root of unity. Let $M=L(\zeta)$. Then the following natural map is injective:

$$
L^{\times} / L^{\times p^{\nu}} \rightarrow M^{\times} / M^{\times p^{\nu}} .
$$

We claim that Proposition 1.5 follows from the previous lemma (which we will prove below). Indeed, let $\mathbf{F}_{\nu}=K_{\wp}(\zeta)$ where $\zeta$ is a primitive $p^{\nu}$ th root of unity. The natural map

$$
K_{\wp}^{\times} / K_{\wp}^{\times p^{\nu}} \rightarrow \mathbf{F}_{\nu}^{\times} / \mathbf{F}_{\nu}^{\times p^{\nu}}
$$

is injective by the previous lemma, so the image of the group generated by the cosets of $q$ and $\sigma(f)$ also has order $p^{2 \nu}$.

It follows that $\left[\mathbf{F}_{\nu}\left(q^{1 / p^{\nu}}, \sigma(f)^{1 / p^{\nu}}\right): \mathbf{F}_{\nu}\right]=p^{2 \nu}$ and we can deduce that

$$
\left[\mathbf{F}_{\nu}\left(q^{1 / p^{\nu}}, \sigma(f)^{1 / p^{\nu}}\right): \mathbf{F}_{\nu}\left(q^{1 / p^{\nu}}\right)\right]=p^{\nu} .
$$

Hence $\sigma(f)^{1 / p^{\nu}}$ has degree $p^{\nu}$ over $\mathbf{F}_{\nu}\left(q^{1 / p^{\nu}}\right)=K_{\wp}\left(B\left[p^{\nu}\right]\right)$, so $\sigma(f)^{1 / p}$ has degree $p$ over $K_{\wp}\left(B\left[p^{\nu}\right]\right)$.

Proof of Lemma 1.7. As a consequence of Hilbert's Theorem 90 we obtain:

$$
H^{1}\left(\operatorname{Gal}(\bar{L} / L), \mu_{p^{\nu}}\right)=L^{\times} / L^{\times p^{\nu}}, \quad H^{1}\left(\operatorname{Gal}(\bar{M} / M), \mu_{p^{\nu}}\right)=M^{\times} / M^{\times p^{\nu}} .
$$

Moreover, the natural map $L^{\times} / L^{\times p^{\nu}} \rightarrow M^{\times} / M^{\times p^{\nu}}$ corresponds to the restriction map in cohomology, which fits in the exact sequence

$$
0 \rightarrow H^{1}\left(\operatorname{Gal}(M / L), \mu_{p^{\nu}}\right) \rightarrow H^{1}\left(\operatorname{Gal}(\bar{L} / L), \mu_{p^{\nu}}\right) \rightarrow H^{1}\left(\operatorname{Gal}(\bar{M} / M), \mu_{p^{\nu}}\right) .
$$

Thus, in order to show that the map is injective, is enough to show that

$$
H^{1}\left(\operatorname{Gal}(M / L), \mu_{p^{\nu}}\right)=0 .
$$

Since $M=L(\zeta)$ where $\zeta$ is a primitive $p^{\nu}$ th root of unity, we can think of $\operatorname{Gal}(M / L)$ as a subgroup of $\left(\mathbb{Z} / p^{\nu} \mathbb{Z}\right)^{\times}$acting on $\mu_{p^{\nu}} \cong \mathbb{Z} / p^{\nu} \mathbb{Z}$ via multiplication, and to finish the proof, we must prove:

LEMma 1.8. $H^{1}\left(G, \mathbb{Z} / p^{\nu} \mathbb{Z}\right)=0$ for any $G \leq\left(\mathbb{Z} / p^{\nu} \mathbb{Z}\right)^{\times}$. 
Statements similar to this one can be found in the literature (see e.g. [Rub99, Lemma 6.1]), but for the convenience of the reader we include a proof of the precise statement needed here.

Proof. For this, let $\psi: G \rightarrow \mathbb{Z} / p^{\nu} \mathbb{Z}$ be a cocycle. We wish to prove that $\psi$ is actually a coboundary. Since $G \leq\left(\mathbb{Z} / p^{\nu} \mathbb{Z}\right)^{\times}, G$ is cyclic, that is, $G=\langle a\rangle$ for some $a$. Moreover, suppose that the order of $G$ is $n_{0}$. Since $\psi$ is a cocycle $\psi(1)=0$ and, inductively, one can show that

$$
\psi\left(a^{t}\right)=\left(a^{t-1}+a^{t-2}+\cdots+1\right) \psi(a)=\left(\frac{a^{t}-1}{a-1}\right) \psi(a) .
$$

Note that $1 /(a-1)$ might not make sense in $\mathbb{Z} / p^{\nu} \mathbb{Z}$, so we also let $a$ be an integer representative of the congruence class, and we write $\left(\left(a^{t}-1\right) /(a-1)\right)$ for the congruence class of $\left(a^{t}-1\right) /(a-1) \in \mathbb{Z}$ modulo $p^{\nu} \mathbb{Z}$.

Note that $n_{0}$, the order of $G$, divides $p^{\nu-1}(p-1)$, the order of $\left(\mathbb{Z} / p^{\nu} \mathbb{Z}\right)^{\times}$. First, suppose that $\operatorname{gcd}\left(n_{0}, p-1\right)>1$. Then $a \neq 1 \bmod p$, since the elements which are congruent to 1 modulo $p$ generate subgroups with order a power of $p$. Since $a \neq 1 \bmod p, a-1 \in\left(\mathbb{Z} / p^{\nu} \mathbb{Z}\right)^{\times}$and it follows that

$$
\text { (a) } \quad \psi\left(a^{t}\right)=\left(\frac{a^{t}-1}{a-1}\right) \psi(a)=\left(a^{t}-1\right) \frac{\psi(a)}{a-1}=a^{t} \frac{\psi(a)}{a-1}-\frac{\psi(a)}{a-1}
$$

with $\psi(a) /(a-1) \in \mathbb{Z} / p^{\nu} \mathbb{Z}$. Hence $\psi$ is a coboundary in this case.

Only the case $n_{0}=p^{\nu-m}$ remains, where $m$ is an integer satisfying $1 \leq$ $m<\nu$. This corresponds to the case $G=\left\{\alpha \in\left(\mathbb{Z} / p^{\nu} \mathbb{Z}\right)^{\times}: \alpha \equiv 1 \bmod p^{m}\right\}$. Thus $a$, the chosen generator of $G$, satisfies $a \equiv 1+u p^{m} \bmod p^{\nu}$, with $u \neq 0 \bmod p$. It suffices to show that $\psi(a) \equiv 0 \bmod p^{m}$ since that will imply that $\psi(a) /(a-1) \in \mathbb{Z} / p^{\nu} \mathbb{Z}$ and we can proceed as in (\$) to prove that $\psi$ is a coboundary. We start with

$$
0 \equiv \psi(1) \equiv \psi\left(a \cdot a^{p^{\nu-m}-1}\right) \equiv \psi(a)+a \cdot \psi\left(a^{p^{\nu-m}-1}\right) \bmod p^{\nu}
$$

and

$$
\psi\left(a^{p^{\nu-m}-1}\right) \equiv\left(\frac{a^{p^{\nu-m}-1}-1}{a-1}\right) \psi(a) \bmod p^{\nu}
$$

thus

$$
0 \equiv \psi(a)+a\left(\frac{a^{p^{\nu-m}-1}-1}{a-1}\right) \psi(a) \bmod p^{\nu} .
$$

It is easy to see that $\left(1+u p^{\eta}\right)^{p^{\kappa}}=1+u^{\prime} p^{\eta+\kappa}$, with $u \equiv u^{\prime} \bmod p$. Hence

$$
a\left(\frac{a^{p^{\nu-m}-1}-1}{a-1}\right)=\frac{a^{p^{\nu-m}}-1}{a-1}-1 \equiv p^{\nu-m}-1 \bmod p^{\nu+1}
$$

and the congruence remains true modulo $p^{\nu}$. Finally, substituting in ( above, we obtain 


$$
0 \equiv \psi(a)+\left(p^{\nu-m}-1\right) \psi(a) \equiv p^{\nu-m} \psi(a) \bmod p^{\nu} .
$$

Therefore, $\psi(a) \equiv 0 \bmod p^{m}$, which concludes the proof of the lemma.

We have thus finished the proof of Theorem 1.1.

2. Example. Let $K=\mathbb{Q}(\sqrt{-11}), p=11$ and set $\tau=(1+\sqrt{-11}) / 2$. We write $\wp$ for the unique prime ideal of $K$ lying above 11, thus the ramification index $e=e(\wp \mid p)$ is 2 . Let $A / K$ be the curve

$$
A: y^{2}+(2 \tau-1) y=x^{3}+\tau x^{2}, \quad j(A)=\frac{-61440-851968 \tau}{11 \cdot 4931},
$$

$\Delta_{A}=-3795-352 \tau, \quad N_{K / \mathbb{Q}}\left(\Delta_{A}\right)=3^{3} \cdot 11^{2} \cdot 3941, \quad N_{K / \mathbb{Q}}(j(A))=\frac{2^{24} \cdot 3^{3}}{11^{2} \cdot 3941}$.

In particular, $t=-v_{\wp}(j(A))=2$. Note that $e=2$ is not divisible by $p-1=10 ; \operatorname{gcd}(p, t)=\operatorname{gcd}(11,2)=1$ and $e p /(p-1)=11 / 5>2=t$.

Hence it remains to check that the representation $\widetilde{\varrho}_{A}: \operatorname{Gal}(\bar{K} / \widetilde{K}) \rightarrow$ $\operatorname{SL}\left(2, \mathbb{F}_{p}\right)$ is surjective. In [Ser72, Proposition 19], J.-P. Serre gives conditions for a subgroup $G$ of $\operatorname{SL}\left(2, \mathbb{F}_{p}\right)$ to be the full group $\operatorname{SL}\left(2, \mathbb{F}_{p}\right)$. We reproduce the result here for the reader's convenience:

Proposition. Suppose $p \geq 5$ and the following hypotheses are satisfied:

(1) the subgroup $G$ contains a matrix $s_{1}$ such that $\operatorname{Tr}\left(s_{1}\right)^{2}-4 \operatorname{det}\left(s_{1}\right)$ is a nonzero quadratic residue modulo $p$, and $\operatorname{Tr}\left(s_{1}\right) \neq 0 \bmod p$;

(2) $G$ contains a matrix $s_{2}$ such that $\operatorname{Tr}\left(s_{2}\right)^{2}-4 \operatorname{det}\left(s_{2}\right)$ is not a quadratic residue modulo $p$, and $\operatorname{Tr}\left(s_{2}\right) \neq 0 \bmod p$;

(3) $G$ contains a matrix $s_{3}$ such that $u=\operatorname{Tr}\left(s_{3}\right)^{2} / \operatorname{det}\left(s_{3}\right)$ is not $0,1,2$ or 4 modulo $p$ and $u^{2}-3 u+1 \neq 0 \bmod p$.

Then $G$ is the full group $\mathrm{SL}\left(2, \mathbb{F}_{p}\right)$.

Let $G<\mathrm{SL}\left(2, \mathbb{F}_{p}\right)$ be the image of the representation $\widetilde{\varrho}_{A}$. Let $S_{A}$ denote the set of all prime ideals of $K$ such that $A$ has bad reduction. $S_{A}$ is the set of prime ideals which divide $\Delta_{A}$, i.e. $S_{A}=\{3,11,3941\}$. Then, for every $\nu \notin S_{A} \cup\{\wp\}$, the image via $\widetilde{\varrho}_{A}$ of a Frobenius element $\pi_{\nu} \in \operatorname{Gal}(\bar{K} / \widetilde{K})$ is a matrix that we also denote by $\pi_{\nu}$ such that:

(1) $\operatorname{Tr}\left(\pi_{\nu}\right) \equiv a_{\nu} \bmod p$ where $a_{\nu}$ is the trace of the Frobenius automorphism of $A$ at the place $\nu$;

(2) $\operatorname{det}\left(\pi_{\nu}\right) \equiv \mathbf{N}(\nu) \bmod p$.

In order to conclude that $G=\operatorname{SL}\left(2, \mathbb{F}_{p}\right)$ we exhibit three Frobenius elements $s_{1}, s_{2}, s_{3}\left(s_{3}=s_{2}\right)$ that satisfy the conditions in the Proposition above. The trace of the Frobenius automorphism was calculated using the computer software PARI [Pari00]. 
- The prime number 5 is split in $K$. Let $\nu_{5}$ be one of the prime ideals of $K$ lying above 5 (so $\mathbf{N}\left(\nu_{5}\right)=5$ ). The trace of the Frobenius automorphism is $a_{\nu_{5}}=-1$. Let $s_{1}=\pi_{\nu_{5}}$. Then

$$
\operatorname{Tr}\left(s_{1}\right)^{2}-4 \operatorname{det}\left(s_{1}\right) \equiv(-1)^{2}-4 \cdot 5 \equiv-19 \equiv 5^{2} \bmod 11 .
$$

- The prime number 13 is inert in $K$. Let $\nu_{13}$ be the prime ideal of $K$ lying above 13 (so $\mathbf{N}\left(\nu_{13}\right)=169$ ). The trace of the Frobenius automorphism is $a_{\nu_{13}}=10$. Let $s_{2}=\pi_{\nu_{13}}$. Then

$$
\operatorname{Tr}\left(s_{2}\right)^{2}-4 \operatorname{det}\left(s_{2}\right) \equiv(10)^{2}-4 \cdot 169 \equiv-576 \equiv 7 \bmod 11
$$

and 7 is not a quadratic residue modulo 11 .

- Let $s_{3}=s_{2}$ and let $u=\operatorname{Tr}\left(s_{3}\right)^{2} / \operatorname{det}\left(s_{3}\right) \equiv \frac{100}{169} \equiv 3 \bmod 11$. Then $u^{2}-3 u+1 \equiv 1 \bmod 11$.

Therefore $\widetilde{\varrho}_{A}$ is surjective and all conditions of Theorem 1.1 have been verified, thus the map $\varrho_{A}: \operatorname{Gal}(\bar{K} / \widetilde{K}) \rightarrow \mathrm{SL}\left(2, \mathbb{Z}_{11}[[X]]\right)$ is surjective.

Acknowledgements. I would like to thank David Rohrlich for his dedication as my advisor.

\section{References}

[Bos86] N. Boston, Appendix to [MW86], Compositio Math. 59 (1986), 261-264.

[Deu53] M. Deuring, Die Zetafunktion einer algebraischen Kurve vom Geschlechte Eins, Nachr. Akad. Wiss. Göttingen Math.-Phys. Kl. IIa 1953, 85-94; II, ibid. 1955, 13-42; III, ibid. 1956, 37-76; IV, ibid. 1957, 55-80.

[Deu58] -, Die Klassenkörper der komplexen Multiplikation, in: Enzyklopädie der mathematischen Wissenschaften: Mit Einschluss ihrer Anwendungen, Band I-2, Heft 10, Teil II, Teubner, Stuttgart, 1958.

[KL81] D. S. Kubert and S. Lang, Modular Units, Grundlehren Math. Wiss. 244, Springer, New York, 1981.

[Lan87] S. Lang, Elliptic Functions, 2nd ed., Springer, New York, 1987.

[MW86] B. Mazur and A. Wiles, On p-adic analytic families of Galois representations, Compositio Math. 59 (1986), 231-264.

[Pari00] The PARI Group, PARI/GP, Version 2.1.1, 2000, Bordeaux, available from http://www.parigp-home.de/

[Roh] D. E. Rohrlich, Universal deformation rings and universal elliptic curves, unpublished note (available at his website).

[Roh00a] -, False division towers of elliptic curves, J. Algebra 229 (2000), 249-279.

[Roh00b] —, A deformation of the Tate module, ibid. 229 (2000), 280-313.

[Roh04] -, Modular units and the surjectivity of a Galois representation, J. Number Theory 107 (2004), 8-24.

[Rub99] K. Rubin, Elliptic curves with complex multiplication and the conjecture of Birch and Swinnerton-Dyer, in: Arithmetic Theory of Elliptic Curves (Cetraro, 1997), Lecture Notes in Math. 1716, Springer, Berlin, 1999, 167-234.

[Ser68] J.-P. Serre, Abelian l-adic Representations and Elliptic Curves, W. A. Benjamin, New York, 1968. 
[Ser72] J.-P. Serre, Propriétés galoisiennes des points d'ordre fini des courbes elliptiques, Invent. Math. 15 (1972), 259-331.

[ST68] J.-P. Serre and J. Tate, Good reduction of abelian varieties, Ann. of Math. 88 (1968), 492-517.

Department of Mathematics

Colby College

8800 Mayflower Hill

Waterville, ME 04901, U.S.A.

E-mail: alozano@colby.edu

Received on 6.9.2004

and in revised form on 18.11.2004 